\title{
Parents' perception of their role in the prevention of inadequate consumption of fruit and vegetables among adolescents in South Africa
}

SADJ June 2020, Vol. 75 No. 5 p235 - p240

TE Okagbare ${ }^{1}$, S Naidoo ${ }^{2}$

\section{ABSTRACT}

\section{Introduction}

South African adolescents consume relatively low fruit and vegetables in spite of the development of foodbased dietary guidelines for South Africa.

\section{Aims and objectives}

The aims and objectives of the present study were to investigate parents' perception of their role in the prevention of the health compromising behaviour of inadequate consumption of fruit and vegetables among adolescents in South Africa.

\section{Design}

The study design was qualitative and exploratory and the research strategy used was inductive, deductive and abductive.

\section{Methods}

Using a guiding question schedule data were collected from five focus group interviews. A non-probability purposive theoretical sampling method was utilized and the sample size of 37 determined by theoretical saturation. Data analysis was performed using the grounded theory approach.

\section{Results}

The findings of the study suggest that children should be introduced to fruit and vegetables as early as possible.

\section{Conclusion}

The study recommends that infants should be exposed to the taste of locally available fruit and vegetables fol-

\section{Author affiliations:}

1. Tuweyire E Okagbare: BDS, PG Dip. (Odont), MSc (Odont), PhD Department of Dental Public Health, Faculty of Dentistry, University of the Western Cape, South Africa. ORCID Number: 0000-0002-8263-2866

2. Sudeshni Naidoo: BDS, LDS.RCS, MDPH, DDPH.RCS, MCh $D, P h D$, Dip IRE., DSc, Faculty of Dentistry, University of the Western Cape, South Africa. ORCID Number: 0000-0001-7424-8082

Corresponding author: Tuweyire E Okagbare

Faculty of Dentistry, University of the Western Cape, South Africa.

Private Bag X17, Bellville 7535, South Africa.

Email: teokagbare@gmail.com

Author contributions:

1. Tuweyire E Okagbare: Principal researcher - $60 \%$

2. Sudeshni Naidoo: Co-author \& advisor - $40 \%$ lowed by the gradual introduction of fruit and vegetables into their diet together with a variety of other nutrientdense foods when solid foods are being introduced.

\section{Keywords}

Qualitative research, adolescents, taste buds, inadequate fruit and vegetables, interventions, oral health, South Africa.

\section{INTRODUCTION}

The perception that nutrient-based dietary guidelines are not effective in promoting appropriate diets and healthy lifestyles has motivated a number of countries including South Africa to develop food-based dietary guidelines (FBDGs) in accordance with the recommendation of Food and Agriculture Organisation/World Health Organization (FAO/WHO).

The South African FBDGs were satisfactorily tested for comprehension, appropriateness and applicability in consumer groups of different ethnic backgrounds in both rural and urban areas. ${ }^{1}$ Consuming a diet high in fruit and vegetables is associated with a decreased risk of many chronic diseases, including periodontal disease because fruits and vegetables are good sources of many important nutrients such as potassium, vitamin C, folate, fiber, and numerous phytochemicals. ${ }^{2}$

Even though the composition of the family unit has undergone considerable change in recent decades due to a variety of socio-economic developments, it remains the first learning environment for the child. The influence of the family continues throughout adolescence and indeed throughout the life-course of the individual to varying degrees because parents are powerful role models and influence. ${ }^{3}$ Consumption of fruit/vegetables among adolescents is a healthy behaviour consistently related to higher parental socio-economic status (SES). ${ }^{4}$

Foods high in fat and sugar are cheaper per unit energy when compared with foods rich in protective nutrients such as fruits and vegetables. ${ }^{5}$ This is also the reason why social support correlates positively with fruit/vegetable intake. ${ }^{6}$ 
The most important predictor of fruit and vegetable consumption is suggested to be gender ${ }^{7}$, and this difference is also evident in children as young as four years of age. ${ }^{8}$ A Norwegian study involving adolescents, found that boys ate less fruit and vegetables than the girls because they like them less. However, the reasons for this are yet to be identified. ${ }^{9}$

Rovner et al. ${ }^{10}$ indicate that the availability of fruits and vegetables in the school environment is positively associated with the consumption of these foods by younger learners. A Minnesota study on the other hand, indicates that snack vending machines availability in schools are associated with a decrease in the consumption of fruits among seventh graders. ${ }^{11}$

South African adolescents consume relatively low fruit and vegetables in spite of the development of foodbased dietary guidelines. ${ }^{1}$ There is need to understand the role of specific psycho-social factors associated with low fruit and vegetables consumption among adolescents in South Africa and to provide scientific evidence that may lead to the development of health promotion strategies, targeted interventions that are appropriate and effective from the perspective of parents.

This is because unhealthy lifestyles in adolescence including the low intake of fruit and non-starchy vegetables may predict the occurrence of adult chronic systemic conditions which may include or determine risk for poorer oral/general health status in adults. ${ }^{12}$

The present study was therefore, designed to provide an opportunity to generate new knowledge and improve the understanding of pathways to oral health inequalities among adolescents through the investigation of parents' perception of the psycho-social factors associated with inadequate consumption of fruit and non-starchy vegetables among adolescents in South Africa.

\section{MATERIALS AND METHODS}

\section{Study design and sampling procedure}

The study design was a Double-Layer Design, which had geographic areas as the first layer and the different audiences as the second layer. It was qualitative exploratory and the research strategy used was inductive, deductive and abductive. The sampling method utilized was the non-probability purposive theoretical sampling method.

\section{Profile of participants}

The number of participants recruited was 37 ; mean age 46.3 years $[95 \% \mathrm{Cl}=43.0-49.5]$. They were homogeneous in the sense of shared experience of residing in one community but diverse in terms of their professions.

No attempt was made to achieve a provincially representative sample but in order to ensure that the data was not skewed the study endeavoured to accommodate participation from both the urban and semi-rural areas. This was also the reason why the four racial groupings in South Africa (Black, Coloured, Asian/Indian and White) were recruited and the perspectives of the two major global religious groupings (Christianity and Islam) obtained.

\section{Data collection}

Five focus group interviews of subjects who met the criteria of the study, of being a past/current parent or caregiver to an adolescent were conducted between months of March and November, 2015. The interviews were conducted in venues acceptable and accessible to all the invitees.

The researcher used the following opening question to ensure that the participants meant the inclusion criteria: Please, has any adolescents between the ages of 10 to 19 year lived with you or living with you at the moment?"

Consent was obtained and the participants were then requested to complete a short questionnaire on demographic characteristics.

A semi-structured interview guide developed for this study was used to ensure consistency in data collection from the focus group interviews while at the same time, allowing the interview sessions to be flexible enough to optimize the natural flow of discussion. In order to allow the participants to freely express themselves and reduce the chance of priming the discussion, the interview guide included a series of open-ended questions.

These questions were designed to stimulate discussion among the participants regarding their perception of their role in the prevention of inadequate consumption of fruit and vegetables among adolescents in South Africa. The use of the layperson's language for the benefit of all participants was encouraged so as to reduce the odds of inhibition. The participants took turns. This was to ensure that they all had equal opportunity to contribute to the discussion.

\section{Data analysis}

This study applied the grounded theory methodology in the data analysis. Data analysis of the transcripts began with Open (Substantive) and Axial Simultaneous Coding method (First and Second Cycle coding processes) which was employed right from Initial Coding up to integrated data analysis. A specialist in Community Dentistry was engaged as an independent coder to reduce bias.

\section{Ethical considerations}

This study was approved by the Senate Research Ethics Committee of the University of the Western Cape, South Africa (Ref No. 11/1/55). Details of the sample procedure of this study have earlier been published. ${ }^{13,14}$ In brief, five focus groups interviews (minimum of six participants each) were conducted between the months of March and November, 2015.

Participants were past/current parents or caregivers to adolescents. Pseudonyms were used to ensure confidentiality was maintained throughout the study by not revealing the true identities of the participants. 
The purpose of the study was explained by researcher to the understanding of the participants and were shown the ethical approval for the study before they read and signed the informed consent forms.

Permission to use the audio recorder was obtained from each participant at the commencement of every interview. The participants were informed that their participation in the interview was entirely voluntary and that anyone may decline to answer any questions.

The researcher made it clear that any of them was at liberty to withdraw from the interview at any time without consequences.

\section{RESULTS}

The sample size of 37 which was determined by theoretical saturation included 23 fathers, one grandfather, 10 mothers and three grandmothers. Thirteen of them had tertiary education while 14 had matriculated and 10 did not have matric. Overall, 25 Blacks, seven Coloured, three Indians and two Whites were recruited. Thirty of these participants were Christians while the remaining seven were Muslims.

The results of the data analysis using the grounded theory methodology were articulated in the two substantive categories below:

1. Adolescent inadequate consumption of fruit and vegetables.

2. Parents' perception regarding their roles.

\section{Adolescent inadequate consumption of fruit and vegetables}

\section{The home environment}

The study participants were unanimous regarding the important role of good upbringing of children starting at a very young age and the importance of the home environment in the formation and retention of the healthy behaviour of consuming fruit and vegetables.

There was complete agreement among the participants that a parent's healthy behaviour had a significant effect on their adolescent children's tendency towards that behaviour because parents are powerful role models that their children emulate.

Therefore, parents should be positively exemplary. The participants also, opined that different races eating differently; some consuming more vegetables than others.

Children reject foods that do not taste "nice" such as certain vegetables and they are also choosy. Below are some excerpts from the participants' discussions:

Vegetables and fruits - that's based on the way you grew up. If you grew up eating a lot of vegetables and fruits then you eat a lot.

...these kids when they growing up they ...don't eat fruits or veggies... they are very choosy... ...people from certain cultures eat much more fruit or vegetables and stuff like that...

You have to be a right role model from when the child is small they look up at you as the parent. They imitate everything you do. So, if you do the right stuff the child will remember...

What I can say is with that kids eating behaviour... it depends on the home environment that they were growing up in.

\section{Taming and training the taste buds}

The participants expressed their concern regarding adolescents' preferences mainly on account of the taste of fruit and vegetables in comparison with confectionaries.

If an adolescent grew up eating fruits and vegetables, the adolescents were likely to remain so. Some excerpts below illustrate their concern:

I think with vegetables, some of these ....... are not very nice... they don't taste nice. So, you can't tell a child to eat them because they are not as nice as burgers...

The wife, the mother trained that child from small, they never exposed the child to sweets and that child eats vegetables, that child eat raw food and vegetables with every meal. And for the child that is a normal thing because they don't know anything else. So, all the other cousins come and look, and, they shocked to see the child eating so much vegetables...

\section{Influence of school environment}

The study participants highlighted the significant influence of the school environment on adolescents' attitudes towards the healthy lifestyle of consuming fruit and vegetables as illustrated by the excerpts below:

Now at school they don't sell veggies. I mean the school food kiosk. They sell only sweets and chips, Doritos what do you call them. They sell coke ...no fruit salad, not vegetable salad...

I don't think the schools teach children about the importance of fruits and vegetables. Even the midday meals don't have fruits and veges. They give fruit juice because it can go round when water is added...

\section{Influence of advertisements, marketing, negative mass and social media}

Advertisement and marketing of healthy behaviours and products attract adolescents to such behaviours but nobody advertises fruit and vegetables asserted the participants. Below are some contributions:

...no-one advertises fruits or vegetables like sweet things... what's advertised is what attracts people.

If you advertise a cucumber in TV and you explain the goodness of it and what I'm getting from it, may be, I will consider buying it but you don't see that. Because there is 
no-one that is going to benefit from advertising cucumbers in the TV...

\section{Affordability of fruit and vegetables}

The participants lamented that fruits are not as cheap as sweet things but they also indicated that eating unhealthily is also a matter of priority not affordability only.

Some of their responses were:

May be the affordability. Fruit and vegetables are not very cheap. To buy an apple maybe is three rand. Now fifty cents can buy sweets.

There is some families which are disadvantaged but with families that can afford it, it may be all about priorities. Some people rather buy clothes and other things than buy fruit. You know, they look at fruit, they say it is expensive. But if you compare fruit and a pair of shoes, they will rather go and buy a pair shoes.

\section{Parents' perception regarding their roles}

This section is conceptualised as a multifaceted construct grounded in the interview data and it had the following components:

\section{Parental knowledge}

The participants in the present study appeared to be knowledgeable regarding the benefits of consumption of fruit and vegetables, including the nutritional/health values and the cleansing effects on the oral cavity.

If you eat vegetables and fruits you become healthy because they add vitamins, nutrients, all the carbohydrates, proteins. You grow up healthy. Your teeth will be strong and clean. You have calcium and everything. But if don't take them you are not that healthy.

For example, carrots have a cleaning effect in your mouth but if you don't know that... you will, you keep eating sweets and what not. You leave things that are healthy... so the disadvantage is that you are not eating something that is keeping you alive, that is keeping your sight functional well, that is cleaning you, that is giving you a good body, a good system. You know because some fruits, some vegetables, they help clean your body.

\section{Challenges faced by parents of adolescents}

The challenges parents face include children's general dislike for vegetables, peer pressure and outside influences so parents cannot raise their children alone.

I think we need help as parents with each other to, to raise kids. We can't raise these children alone.

Ah, yea, these days kids don't like, love vegetables. They hate it, whoa.

You see we can try to do our best by our home. Outside, they at school, the other ones, eating those sweet things, chocolates ...so they will share as kids.

\section{DISCUSSION}

While the development of affordable, positive, practical, sustainable and culturally sensitive FBDGs to help South Africans, aged five years and over is commendable, South African adolescents still consume relatively low fruit and vegetables. ${ }^{2}$ This is likely because children as young as four years of age ${ }^{8}$, perhaps much younger have already formed their taste preferences for fruit and vegetables. The main concern of this study was adolescents' preference for sweets and confectionaries mainly on account of the perceived lack of taste of fruit and vegetables.

The important role of good upbringing of children when they are infants and also of the home environment in the formation and retention of the healthy behaviour of consuming fruit and vegetables was highlighted by the present study. There was also the opinion that different races eating differently; some consuming more vegetables than others and that if an adolescent grew up eating fruits and vegetables, the adolescents was likely to continue into their adulthood.

This opinion is supported by a recent study conducted in the United States of America, which found that older Black and White women eat differently although the dietary pattern within each racial group varied considerably depending on factors such age, socio-economic status and neighbourhood. ${ }^{15}$ Apart from different races eating differently, the interview outcome of the present study did not perceive race has a dominant influence on adolescent's attitude towards fruit and vegetables. However, with both acculturation and globalization there are changes in preferences for certain foods and these changes may differ by ethnic groups. For example, in one study, first-generation Asian and Latino adolescents consumed higher fruit and vegetables and lower soda than the American white population. With succeeding generations, the consumption of these items by Asians remains stable. In contrast, fruit and vegetables consumption by Latinos decreased while their soda consumption increases, so that by the third generation their nutrition has become poorer than that of the American white population. ${ }^{16}$

Although, culture is a dynamic construct because it changes over time, it still remains a significant influence over what adolescents consume because of familiarity, and perceived healthfulness. Culture has food beliefs around certain meals and food has indeed, been both an expression of cultural identity and a means of preserving family and community unity. ${ }^{17,18}$ The present study also identified parents' healthy behaviours to have significant effect on their adolescents' tendency towards such behaviours because parents are powerful role models, as their children emulate them. Parents should therefore, be positively exemplary.

There are two main ways parents' modelling can increase a child's consumption of fruit and vegetables. Observation could change behaviours directly or frequent provision of fruit and vegetables at meals could increase the possibility of consumption by promoting liking through increased taste exposure. ${ }^{19}$ Parents therefore, need to be active and positive role models by eating a wide variety of foods and fruit/vegetables regularly. Parents should also ex- 
pose their children to fruit and vegetables through movies, books, or gardening to stimulate their interest in them. ${ }^{20}$ Consistent with previous studies the present study highlighted the significant influence of the school environment on adolescents' attitudes towards the healthy lifestyle of consuming fruit and vegetables. ${ }^{1,10,11}$ Schools should not only teach the value of fruit and vegetables but should encourage their availability within the school premises in favour of snack venting machine ${ }^{10}$ and also, include them in midday meals where such are provided. ${ }^{21}$

Adolescents are continuously exposed to a constant barrage of advertisements for sweetened drinks, fast foods and high-caloric snacks on television commercials. ${ }^{22}$ The parents in this study continually referred to the pervasiveness of fast foods in today's daily life. Another area of concern is that fruits are not as cheap as sweet foods although it was also recognized that eating healthy is a matter of priority not affordability only. In situations where affordability is an issue, parents should be creative; preparing attractive and tasty meals using vegetables. ${ }^{21}$ Vegetables are even more likely to provide optimum nutrients and protection because they are usually prepared fresh for meals preferably immediately after harvest, especially, in the rural areas; unlike fruits that are often eaten many days after harvest. Availability and lower cost of vegetables when compared with fruits that are also highly contingent on seasonal fluctuations is therefore, a great advantage. ${ }^{23}$

The parents in the present study appeared to be knowledgeable regarding the benefits of consuming fruit and vegetables. The perception of some of them regarding adolescent inadequate fruit and vegetables consumption was that in spite of the nutritional/health values and the cleansing effects on the oral cavity, children of this age do not like consuming fruit and vegetables because they do not taste nice when compared to the fast foods that are available.

This confirms the assertion of Vorster et al. that South African adolescents consume relatively low fruit and vegetables despite the development of food-based dietary guidelines. ${ }^{2}$ This situation is partly dependent on how the adolescent was brought up and that it may improve if parents consumed fruit and vegetables regularly at home. Besides gender, ${ }^{7}$ parents' fruit and vegetable consumption is one of the strongest predictor of a child's consumption of these foods because a parent's healthy behaviour has significant effect on their adolescents' tendency towards that behaviour. ${ }^{3}$ Whereas some parents understand quite well what they should be teaching and feeding their children, they do not know how to encourage their children to eat healthily and avoid unhealthy behaviours. They lack practical strategies on how to achieve this. ${ }^{24}$

Infants are known to naturally have intrinsic preferences towards certain taste qualities and dislikes for others. They are also choosy and prefer sweet-tasting foods and reject foods that do not taste nice such as certain vegetables. ${ }^{25}$ They are also reluctant to try new foods. A reluctance to try new foods is called neophobia. ${ }^{20}$ Neophobia seems to be minimal around the age of 140-180 days, so infants may be more willing to try new foods at this age ${ }^{26}$, making it the ideal time to help them acquire a taste for vegetables.

However, neophobia can often be overcome in children who have positive role models. Children are more likely to try unfamiliar foods if they have observed someone else eating them, parents (and sometimes siblings). ${ }^{27}$ When parents take a bite of their children's food and show signs of delight or enjoying it, children are more likely to try the food. ${ }^{19}$ Parents therefore, may see better results from offering their children a variety of healthy foods containing fruit and vegetables from a young age and also from repeated exposures to these foods even if the child does not like them at first. ${ }^{27}$

It is important to note here that fruit juice are not as healthy as solid or whole fruits and therefore should be discouraged. Solid or whole fruits not only contain less sugars (approximately 35\% less sugar), the intrinsic sugars in them are healthier than the extrinsic sugars produced when cell membranes rupture during the juicing process. Solid or whole fruits also provide a lot more fibres and nutrition usually lost during the juicing process. $^{28}$

Some of the parents reported that allergies to certain fruits and vegetables could be one of the reasons why they are not eaten. While this claim may be true, it is uncommon. A food allergy is an abnormal immune response to food. The signs and symptoms may range from mild to severe which may include itchiness, swelling of the tongue, vomiting, diarrhea, hives, trouble breathing, or low blood pressure. This typically occurs within minutes to several hours after exposure. When the symptoms are severe it is known as anaphylaxis. ${ }^{29}$ Food intolerance (e.g. lactose intolerance) and food poisoning are different from food allergy. A type of food allergy known as oral allergic syndrome is characterize by a cluster of allergic reactions in the mouth in response to eating certain (usually fresh) fruits, nuts, and vegetables that typically develops with hay fever. ${ }^{30}$

It is pertinent to state here that WHO Fruit and Vegetable Promotion Initiative had concluded that up to 2.7 million lives could be saved annually with adequate fruit and vegetables consumption, and that a low intake of fruit and vegetables is among the top 10 identified risk factors for global mortality. Promoting adequate (>400 g/d) consumption of fruit and vegetables should therefore be a key dietary goal. ${ }^{31}$ Most adolescent oral health behaviours are initiated in infancy and childhood and are likely to track into adulthood with their health implications. ${ }^{32}$

As previous documented ${ }^{1}$ it takes a whole community including the schools and on-going efforts of parents to provide the enabling environment for the development and retention of the healthy behaviour of consuming fruit and vegetables. This is not a task for one parent alone and here lies the huge challenge. However, findings of the present study should be considered in the light of the following limitations. The generalizability of its findings is limited by the qualitative nature of the study because of the use of non-probability, purposive sampling strategy and also because of the small number of research participants. 


\section{CONCLUSION}

Despite the limitations stated above the conclusion of the study is pertinent and weighty. It suggests that targeting South Africans five years and older has not been adequately effective in promoting appropriate consumption of fruit and vegetables. The study therefore, recommends the introduction of infants as early as possible to the taste of locally available fruit and vegetables as well as the gradual introduction of these fruit and vegetables and a variety of other nutrient-dense foods when solid foods are being introduced, in order to promote their liking through increased taste exposure.

This practice has the potential to facilitate the mitigation of the adolescent unhealthy behaviour of inadequate fruit and vegetables consumption. In addition, parents and schools should provide enabling environments that encourage consumption of fruit and vegetables. The study also suggests future investigation of the effects of the introduction of infants very early to the taste of locally available fruit and vegetables as well as the gradual introduction of these fruit and vegetables in their meals when solid foods are being introduced on adolescent healthy behaviour of fruit and vegetables consumption.

\section{References}

1. Vorster HH, Love P, Browne C. Development of food-based dietary guidelines for South Africa - the process. S Afr J Clin Nutr. 2001; 14: 3-6.

2. Centers for Disease Control and Prevention. Strategies to Prevent Obesity and Other Chronic Diseases: The CDC Guide to Strategies to Increase the Consumption of Fruits and Vegetables. Atlanta: U.S. Department of Health and Human Services; 2011

3. Fostera MA, Lambertb R, Abbott-Shima M, Mccartya F, Franze S. A model of home learning environment and social risk factors in relation to children's emergent literacy and social outcomes. Early Child Res Q. 2005; 20: 13-36.

4. Friestad C, Klepp K. Socioeconomic status and health behaviour patterns through adolescence: results from a prospective cohort study in Norway. Eur J Public Health. 2006; 16: $41-7$.

5. James WPT, Nelson M, Ralph A, Leather S. Socioeconomic determinants of health: the contribution of nutrition to inequalities in health. Br Med J. 1997; 314: 1545 - 8.

6. Watt RG, Sheiham A. Dietary patterns and changes in inner city adolescents. J Hum Nutr and Diet. 1996; 9: 451-61.

7. Friel S, Newell J, Kelleher C. Who eats four or more servings of fruit and vegetables per day? Multivariate classification tree analysis of data from the 1998 Survey of Lifestyle, Attitudes and Nutrition in the Republic of Ireland. Public Health Nutr. 2005; 8: 159-69.

8. Wardle J, Guthrie CA, Sanderson S, Rapoport, L. Development of the children's eating behaviour questionnaire. J Child Psychol Psychiatry. 2001; 42: 963-70.

9. Bere E, Brug J, Klepp Kl. Why do boys eat less fruit and vegetables than girls? Public Health Nutr. 2008; 11: 321-5.

10. Rovner AJ, Nansel TR, Wang J, lannotti RJ. Foods sold in school vending machines are associated with overall student dietary intake. J Adolesc Health. 2011; 48: 13-9.

11. Kubik MY, Lytle IA, Hannan PJ, Perry CL, Story M. The association of the school food environment with dietary behaviours of young adolescents. Am J Public Health. 2003; 93: 1168-73.

12. World Health Organisation. Ten facts about chronic diseases. Geneva: WHO. 2005.

13. Okagbare TE, Naidoo S. Parents' perception of psychosocial factors, health compromising behaviours and oral health among adolescents in South Africa. SADJ. 2018; 73: 221-7.

14. Okagbare TE, Naidoo S. Parental participation in the influencing of the unhealthy behaviour of adolescents in their excessive consumption of free sugars: a qualitative study. SADJ. 2018; 74: 165-70.

15. Wenjun Li, Youssef G, Procter-Gray E., et al. Racial differences in eating patterns and food purchasing behaviors among urban older women. J Nutr Health Aging. 2017; 21: 1190-9.

16. Allen ML, Elliott MN, Morales LS, Diamant AL, Hambarsoomian K, Schuster M.A. Adolescent participation in preventive health behaviours, physical activity, and nutrition: differences across immigrant generations for Asians and Latinos compared with Whites. Am J Public Health. 2007, 97: 337-43.

17. Bruss MB, Morris JR, Dannison LL, Orbe MP, Quitugua JA, Palacios RT. Food, culture, and family: exploring the coordinated management of meaning regarding childhood obesity. Health Commun. 2005; 18: 155-75.

18. Macarthur LH, Anguiano R, Gross KH. Are household factors putting immigrant Hispanic children at risk for becoming overweight: a community-based study in eastern North Carolina. J Community Health. 2004, 29: 387-404.

19. Wardle J, Cooke L. Genetic and environmental determinants of children's food preferences. Br J Nutr. 2008, 99: 15-21.

20. Gibson EL, Kreichauf S, Wildgruber A., et al. A narrative review of psychological and educational strategies applied to young children's eating behaviours aimed at reducing obesity risk. Obes Rev. 2012, 13: 85-95.

21. Krølner R, Rasmussen M, Brug J, Klepp K, Wind M, Due P. Determinants of fruit and vegetable consumption among children and adolescents: a review of the literature. Part II: qualitative studies. Int J Behav Nutr Phys Act. 2011; 8: $1479-5868$.

22. Duijster D, De Jong-Lenters M, Verrips E, van Loveren C. Establishing oral health promoting behaviours in children - parents' views on barriers, facilitators and professional support: a qualitative study. BMC Oral Health 2015, 15: 157-70.

23. Love P, Sayed N. Eat plenty of fruit and vegetables every day. S Afr J Clin Nutr. 2001; 14: $24-32$.

24. Schwartz C, Scholtens P, Lalanne A, Weenen H, Nicklaus S. Development of healthy eating habits early in life: review of recent evidence and selected guidelines. Appetite 2011; 57: 796-807.

25. EUFIC Food Today. Tastes differ - how taste preferences develop 2011; Available from: http://www.eufic.org/article/en/ page/FTARCHIVE/artid/how-taste-preferences-develop/ [Accessed 25/03/2016].

26. Mennella JA, Beauchamp GK. The early development of human flavor preferences. In Capaldi ED. (ed.). Why we eat what we eat: the psychology of eating. Washington, DC: American Psychological Association, 1996.

27. EUFIC Food Today. Encouraging young children to eat different vegetables. 2010. Available from: http://www.eufic.org/ article/en/page/FTARCHIVE/artid/Encouraging-young-children-eat-different-vegetables/[Accessed 25/03/2016].

28. Crowe KM, Murray E. Deconstructing a fruit serving: comparing the antioxidant density of select whole fruit and $100 \%$ fruit juices. J Acad Nutr Diet. 2013; 113: 1354 -8.

29. National Institute of Allergy and Infectious Diseases. Food Allergy: an overview 2012; Available from: https://www.niaid. nih.gov/topics/foodAllergy/Documents/foodallergy.pdf [Accessed 27-03-2016].

30. Kelava N, Lugović-Mihić L, Duvanćić T, Romić R. Šitum, M. Oral allergy syndrome - the need of a multidisciplinary approach. Acta Clin Croat 2014; 53:210-19.

31. WHO. Fruit and Vegetable Promotion Initiative-report of the meeting, Geneva, 25-27 August 2003 WHO Geneva. http://www.who.int/dietphysicalactivity/publications/f\&v promotion_initiative_report.pdf. [Accessed 19/09/2019].

32. Gillman, M.W., Rifas-Shiman, S.L., Frazier, A.L., et al. Family dinner and diet quality among older children and adolescents. Arch. Fam. Med. 2000; 9: 235-40. 